\title{
Management in production: from unobserved to observed
}

\author{
Thomas P. Triebs ${ }^{1} \cdot$ Subal C. Kumbhakar ${ }^{2}$
}

Published online: 4 April 2018

(c) The Author(s) 2018

\begin{abstract}
Are productivity estimates good proxies for unobserved management? And, does management affect production in a neutral and monotonic fashion as assumed by these proxies? We use Bloom and Van Reenen's management data to show that two popular proxies, fixed effects and inefficiency scores, correlate with observed management practices. We find that the correlations are positive but weak. Also, management explains only a fraction of the proxies' variances. The data rejects the assumptions of neutrality and monotonicity. Last, our results suggest that management has characteristics both of a technology and an input.
\end{abstract}

Keywords Management practice $\cdot$ production functions $\cdot$ stochastic frontier analysis $\cdot$ semiparametric models

JEL classification Nos: D24 $\cdot$ C14 $\cdot$ M11

\section{Introduction}

Economists often use productivity estimates as proxies for unobserved management, either to control for omitted variable bias or to measure management at the firm level. For instance, Nickell (1996) estimates the effect of competition on firm productivity and assumes that productivity estimates serve as proxies for management quality, among other things. As management is usually unobserved, the correlation between management and productivity estimates ${ }^{1}$ is assumed. Using the management data produced by Bloom and Van Reenen (2007) we are able to test this assumption empirically. We ask whether two popular productivity estimates, firm fixed effects and inefficiency scores, correlate with observed management practices. Also, we ask whether the assumptions underlying their use, neutrality and monotonicity, are valid.

The use of inefficiency estimates as proxies for management goes back at least to Farrell (1957). His model

Thomas P. Triebs

t.triebs@1boro.ac.uk

1 School of Business and Economics, Loughborough University, Loughborough LE11 3TU, UK

2 Department of Economics, State University of New York, Binghamton, NY 13902, USA treats the production function as a best-practice frontier and the difference between actual production and best practice as inefficiency. For given inputs and outputs he attributes estimates of technical inefficiency to bad management ( $p$. 261):

[...] the technical efficiency of a firm or plant indicates the undisputed gain that can be achieved by simply 'gingering up' the management $[\ldots]$.

Farrell's model sets management apart from the conventional inputs of capital and labor in the sense that inefficiency indicates the potential for management to increase output for given conventional inputs, implying that management is a technology. Put differently, unlike conventional inputs, management alone cannot produce. Nuthall (2009, p. 413) expresses this as follows: “... the key factor in the management of land, labor and capital is the management ability applied." On the other hand, Mundlak (1961)'s concern is about omitted variable bias when the management input is unobserved. His model uses firm fixed effects as proxies for management. The model requires panel data and the assumption that management is timeinvariant. We empirically test whether the two proxies, inefficiency and fixed effects, correlate with observed

\footnotetext{
$\overline{1}$ A complication in testing the relation between management and productivity is that the latter is unobserved, too. Productivity is usually estimated from observed input and output quantities.
} 
management. Both proxies assume that management is a productivity shifter and that better management always increases productivity. Put differently, management affects productivity neutrally and the relation between management and productivity is monotonic. Together these assumptions suggest that management is a technology rather than an input. $^{2}$ To empirically test these two assumptions we apply semiparametric technology models where the influence of management can be non-neutral as well as non-monotonic.

A semiparametric model is more general than the parametric linear model Bloom and Van Reenen (2007) use to correlate management with total factor productivity. Their model takes a log-linearized Cobb-Douglas form with management $z$ added in an ad hoc fashion: In $y_{i}=\alpha_{0}+z^{\prime} \alpha$ $+\operatorname{In} x^{\prime} \beta$. They find that management positively correlates with total factor productivity (TFP), $\alpha$ is statistically significant. The parametric linear model helps them validate their survey measure but our investigation of the goodness of productivity as a proxy for management requires a more flexible model. For instance, the use of TFP as a proxy for management requires that TFP increases in management everywhere and not just at the mean. Also, with the parametric linear model, whether management $z$ is a technology depends on the interpretation of the estimated coefficient $\alpha$. Inconsistent with the management as technology interpretation, management alone can produce positive output. The semiparametric models we apply (Robinson 1988; Li et al. 2002) make the technology explicitly management specific: $f^{\mathrm{z}}(x)$, i.e., the production function parameters vary with management. Semiparametric models strike a balance between a standard parametric structure for the conventional inputs and a nonparametric structure for management. The nonparametric part makes the model fully flexible in regard to the effect of management on production and thereby accommodates our theoretical ignorance about how management affects the technology. As the model does not impose the assumptions of neutrality and monotonicity we can test them. Finally, the model allows us to express characteristics of the production function like returns to scale as functions of management.

We find that the correlation between fixed effects or inefficiency scores and management is positive but weak: the largest correlation coefficient is 0.35 . Also, we find that management explains at most $5 \%$ of the variance in the proxies. Firms and policy makers use productivity or inefficiency estimates to assess potential performance improvements through better management. The scope for

\footnotetext{
2 It is conceivable that management is also a conventional input, i.e., a choice variable. Bloom et al. (2012) refer to these alternatives as "management as a technology" and "management as a factor", respectively. They test these alternatives and their empirical results support the theory that management is a technology. But they find that management has some features of a conventional input as well.
}

such improvements might be much lower than suggested by the face values of the proxies. Despite the effect of management on productivity not being strictly neutral, the neutral component gives a relationship between total factor productivity and management that is mostly increasing. Thus, productivity estimates are suitable for ranking firms on management practices but not for quantifying unobserved management practices.

This paper is organized as follows. Section 2 introduces the empirical models that we apply. Section 3 introduces the data. Section 4 gives the results and Section 5 concludes.

\section{Empirical models}

First, we introduce two popular productivity estimates that are also used as proxies for unobserved management. Generally, when management is unobserved it is approximated by $A$, a neutral shift variable outside the production function $f(\cdot)$ :

$$
y=A f(x) \text {, }
$$

where $y$ is output and $x$ is conventional input, e.g., capital and labor. We omit firm and time indices where they are not necessary. In this model, management is a neutral technology-shifter and more management is always better. Neutrality implies, that management is fully separable from the conventional inputs. It does not interact with them, no matter what functional form we choose for $f(\cdot)$. Separability allows us to estimate management when it is unobserved. There are different ways to obtain $A$. One way is to capture $A$ with firm fixed effects as in Mundlak (1961). We replace $A$ with firm fixed effects $\alpha_{\mathrm{i}}$ and append a classical error term $v$ to obtain a standard fixed effects model:

$y_{i t}=\alpha_{i}+f\left(x_{i t}\right)+v_{i t}$,

where $i$ identifies firms and $t$ identifies time. Estimation requires panel data. The fixed effects are proxies for management to the extent that management is timeinvariant. They also capture all other unobserved, timeinvariant factors. In an alternative model, $A$ represents production inefficiency (a random variable). If $A$ is both firm- and year-specific, we can write it as

$$
A_{i t}=A_{0} \frac{A_{i t}}{A_{0}} \equiv A_{0} e^{-u_{i t}}, \quad u_{i t} \geq 0,
$$

where

$$
A_{0} \equiv \max _{i, t}\left\{A_{i t}\right\}, \quad \frac{A_{i t}}{A_{0}} \equiv e^{-u_{i t}} \leq 1
$$

Note that this model can also be estimated in a crosssection. Plugging (3) into (1), taking logarithms, and appending a classical error term $v$, we obtain the standard 
stochastic frontier (SF) production model (Aigner et al. 1977; Meeusen and van den Broeck 1977), viz.,

$\ln y_{i t}=\alpha_{0}+\ln f\left(x_{i t}\right)+v_{i t}-u_{i t}$,

where

$\alpha_{0}=\ln A_{0}$.

The interpretation of this model is that $\ln y_{i t}^{*}=\alpha_{0}+$ $\ln f\left(x_{i t}\right)+v_{i t}$ is the best-practice stochastic frontier technology and inefficiency is the difference $\ln y_{i t}^{*}-\ln y_{i t}$. Only fully efficient firms operate on the frontier. An inefficient firm produces $100\left(\exp \left(u_{i t}\right)-1\right)$ percent less output compared to an efficient firm using the same amount of inputs. This interpretation assumes that it is feasible for any firm to improve its management to the highest level, i.e., attain the best management $A_{0}$. To separate the combined error term $\varepsilon=v-u$, we require assumptions on the distribution of the individual errors. The standard assumptions are

$v_{i t} \sim N\left(0, \sigma_{v}^{2}\right)$,

$u_{i t} \sim N^{+}\left(0, \sigma_{u}^{2}\right)$.

Identification of $u$ is possible when we assume that the distribution of $v$ is symmetric, e.g., normal, but the distribution of $u$ is asymmetric, e.g., half-normal (Jondrow et al. 1982).

As we observe management we can ask whether estimates of fixed effects and inefficiency are good proxies for management by correlating them with management: we regress the productivity estimates on management using the OLS estimator. To better compare inefficiency and fixed effects we estimate inefficiency to be time-invariant and adjust for the intercept. Thus, $\alpha_{i}$ from (2) is equivalent to $\alpha_{0}$ $-u_{i}$ from (5) if $u$ is time-invariant. We control for country and industry fixed effects by mean transforming the variables before estimation, i.e., measurement is in terms of deviations from country-industry means. For the fixed effects model in (2) and the stochastic frontier model in (5) the functional form is translog.

Above, we showed that when management is unobserved, productivity estimates can serve as proxies. As we observe management we can include it into models of production in a way that allows us to test the assumptions of monotonicity and neutrality. First, we extend the stochastic frontier model to include management directly. One way to include management in (5) is to relax the mean zero assumption for $u$ and allow exogenous influences on $u$ using a truncated normal distribution. ${ }^{3}$ We make both the mean and the variance of the inefficiency distribution functions of

\footnotetext{
${ }^{3}$ We used the Stata code made available by Hung-Jen Wang on his web site.
}

management by modifying the assumptions in (7) as follows:

$$
\begin{aligned}
v_{i t} & \sim N\left(0, \sigma_{v}^{2}\right), \\
u_{i t} & \sim N^{+}\left(\mu_{i t}, \sigma_{i t}^{2}\right), \\
\mu_{i t} & =z_{i t} \delta, \\
\sigma_{i t}^{2} & =\exp \left(z_{i t} \gamma\right), \\
\sigma_{v}^{2} & =\exp \left(z_{i t} \lambda\right) .
\end{aligned}
$$

From the estimates of this model we can calculate the marginal effects of management on the mean, $\partial u / \partial z$, and the variance $\partial \sigma^{2} / \partial z$ of the inefficiency distribution. The intuition for the variance of $u$ is that it captures production uncertainty, e.g. better management correlates with lower production uncertainty. Note that as we have a single $z$ variable $-\partial u \partial z=\partial \ln y / \partial z$. Wang (2002) shows that the parametrization in (8) allows the effects of $z$ to be non-monotonic, i.e., management can increase or decrease inefficiency. This is useful, because it allows us to test the monotonicity assumption implied by the proxies. ${ }^{4}$

The stochastic frontier model shifts the production frontier with $u$, which in turn can be a function of management if management is observed. A more straightforward way to shift the production function with management is to allow management to affect the technology in a fully flexible way. To represent this flexibility we write

$y=f^{z}(x)$

where the superscript $z$ indicates that the production function is different for different values of management. To motivate this flexible model, we return to (1) and make $A(\cdot)$ a function of $z$. We can write the model as

$\ln y_{i t}=\ln A\left(z_{i t}\right)+\ln f\left(x_{i t}\right)+v_{i t}$

where $v_{i t}$ is the noise term. This is the semiparametric partially linear (PL) model proposed by Robinson (1988) where $A$ is completely unspecified (nonparametric) but the $x$ variables are specified as usual in a log-linearized form. Here $x$ and $z$ are treated differently in the sense that the functional form for $x$ is parametric but it is nonparametric for $z$. Nested in (10) is the standard form $\ln y_{i}=\alpha_{0}+z^{\prime} \alpha+$ $\ln x^{\prime} \beta$ where $f(\cdot)$ is Cobb-Douglas and $A$ a linear function of

\footnotetext{
${ }^{4}$ The models in (7) and (8) do not separate time-invariant firm heterogeneity from inefficiency leading to an overestimate of true inefficiency in most cases. But as in our sample observed management is largely time-invariant we do not include firm fixed effects in the inefficiency model.
} 
z. Li et al. (2002) generalized the PL model and allowed all parameters to be functions of the $z$ variable: ${ }^{5}$

$\ln y_{i t}=\alpha\left(z_{i t}\right)+\ln x^{\prime} \beta\left(z_{i t}\right)+v_{i t}$.

In this semiparametric smooth coefficient (SPSC) model $z$ affects productivity both neutrally and non-neutrally. The neutral effect is captured by $\alpha\left(z_{i t}\right)$ and the non-neutral effects are captured via the inputs $\beta\left(z_{i t}\right)$. We think of these different effects as channels through which management operates. The PL model in (10) constrains the effects via the inputs to zero. Again, equations (10) and (11) are not linear in parameters since both $\alpha$ and $\beta$ are nonparametric functions of $z$, which are not necessarily linear. The combination of being parametric in $x$ and nonparametric in $z$ makes the function semiparametric. Importantly, this difference captures the economic difference between the conventional inputs of capital and labor and the non-conventional management input.

Technically, these semiparametric models strike a balance between precision and robustness (Robinson 1988). Although fully parametric models are very precise they suffer from possible functional form mis-specification. Nonparametric models are robust but inefficient as they suffer from the curse of dimensionality problem. However, for SPSC models with a single $z$ variable, like ours, the curse of dimensionality is not important ( $\mathrm{Li}$ et al. 2002). Also, the parametric structure makes the semiparametric model less sensitive to outliers than the fully nonparametric model. Appendix A gives details on the estimation of the SPSC model, including outlier detection, and the construction of bootstrapped confidence intervals. For details on the estimation of the PL model see Robinson (1988). An important benefit of the semiparametric models is that they allow us to test the assumptions of neutrality and monotonicity for the influence of management. We test neutrality by testing the Null of the PL model, which restricts the management influence to be neutral, against the SPSC model, which allows for non-neutral effects. Li and Racine (2010) propose the test statistic we use. To test the assumption of monotonicity we visually analyze graphs that plot management-dependent total factor productivity estimates against management for different models. Finally, by testing the Null of a parametric linear model including management against the PL model, using the test by Hsiao et al. (2007) we can also assess whether the neutral effect of management is linear or not.

\footnotetext{
5 Although (10) is nested in (11) the estimation strategies are very different. For details compare Robinson (1988) and Li et al. (2002).
}

\section{Data}

In their seminal paper Bloom and Van Reenen (2007) introduce a survey tool to consistently measure firm-level management practices across countries and industries. Our data is from Bloom and Van Reenen (2010). ${ }^{6}$ The data is for medium-sized manufacturing firms, for a number of countries, and for the years 2002 to $2010 .^{7}$ The number of firmyear observations is 6349 . The panel is highly unbalanced. The average number of observations by firm is 2.4. The firms were surveyed on their management practices using a practice evaluation tool developed in collaboration with a leading management consulting firm. The tool defines and scores 18 separate management practices. Each practice was scored using several questions. The responses were scored from 1 (worst) to 5 (best). Our management variable is the unweighted average across the 18 practices; like Bloom and Van Reenen (2007) we treat it as effectively continuous. Bloom and Van Reenen (2007, p. 1386) state that their measure of management practice captures "organizational capital" rather than employees' raw ability or skills. Therefore, the data should capture the quality of management not managers. All individual questions and example responses are listed in Bloom and Van Reenen (2006). The questions are also reproduced in Appendix B. We only include privately owned firms. We drop the ownership categories "government" and "other". We include the 725 observations that have no value for the ownership variable. The management data is matched with standard accounting data at the firm level. Output $Y$ is measured as sales in current US dollars. Capital input $K$ is tangible fixed assets in current US dollars and labor input $L$ is number of employees. Table 1 provides summary statistics for our full sample. As the semiparametric models are sensitive to outliers we apply an outlier screen for these models only (see Appendix A for details).

\section{Results}

\subsection{Do productivity estimates correlate with observed management?}

To investigate whether estimates of fixed effects and inefficiency are good proxies for unobserved management we regress their estimates against observed management (and a constant). Figure 1 plots lines of best linear fit including 95\% prediction intervals and the raw data. The correlations

\footnotetext{
${ }^{6}$ The data is available at worldmanagementsurvey.org.

7 The countries are Argentina, Australia, Brazil, Canada, Chile, China, France, Germany, United Kingdom, Greece, Italy, Japan, Poland, Portugal, Republic of Ireland, Sweden, and the United States.
} 
in the two panels have opposite signs, because fixed effects represent efficiency whereas the stochastic frontier models estimate inefficiency. The two proxies themselves are highly correlated. The correlation coefficient between the estimates of fixed effects and time-invariant inefficiency is -0.89 .

The OLS coefficient estimates for management are 0.34 and -0.18 for fixed effects and inefficiency scores, respectively. For both regressions the coefficients are significant at a $1 \%$ level. To compare the magnitudes of the responses, a one point increase in the management index (which is roughly two standard deviations) implies an increase in productivity as measured by fixed effects of roughly $50 \%$ of a standard deviation but only $39 \%$ of a standard deviation for the inefficiency scores. A further test for the goodness of the proxies is that the residuals from the second stage regressions should not correlate with management. At standard levels of statistical significance, this is true for the fixed effects but not the inefficiency score residuals. However, there is no economically significant correlation between management and the residuals for either proxy.

Despite these positive correlations, management explains little of the variance in the proxies. The R-squares are 0.057 and 0.035 for the fixed effects and inefficiency models, respectively. Remember that our hypothesis is that the proxies are management, implying that the $\mathrm{R}$-squares equal

Table 1 Summary statistics

\begin{tabular}{lllll}
\hline & Mean & St. Dev. & Min. & Max. \\
\hline Sales (US\$) & 240,047 & 795,196 & 7 & $16,067,543$ \\
Capital (US\$) & 58,203 & 211,438 & 2 & $4,266,051$ \\
Labor (\#) & 869 & 3016 & 1 & 65,682 \\
Management score & 3 & 1 & 1 & 5 \\
Observations & 6349 & & & \\
\hline
\end{tabular}

a Fixed effects

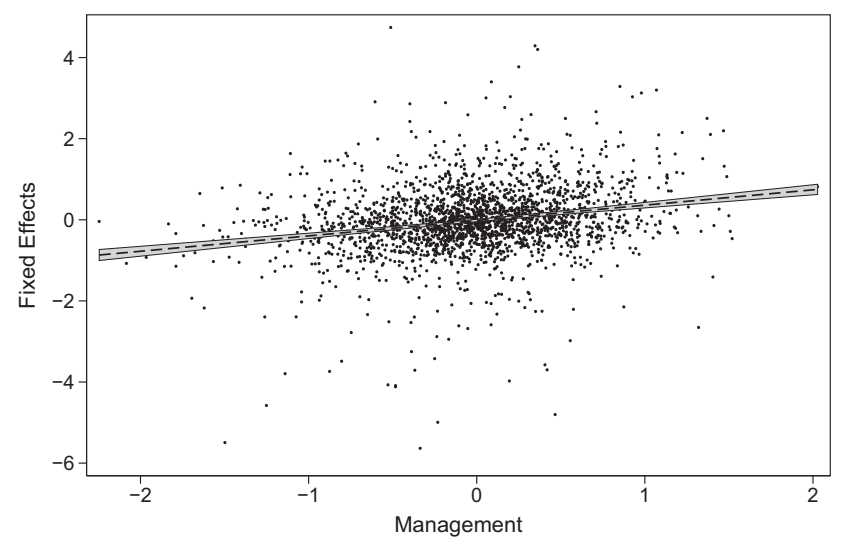

1. These low R-squares are consistent with the fact that adding the management variable to a Cobb-Douglas specification of a conventional production function only increases R-squared from 0.665 to 0.672 (remember that our data is transformed around industry and country means).

When comparing fixed effects and inefficiency scores as proxies for management the former perform better but require panel data. Absolutely, fixed effects and inefficiency scores are both poor proxies for management as management explains only a small fraction of the proxies' variation. This is no surprise as the proxies also capture many other unobserved factors of production.

\subsection{Is the influence of management neutral and monotonic?}

Above we investigated whether productivity estimates correlate with observed management. In this section, to test whether total factor productivity is always increasing in management and whether management affects productivity neutrally or non-neutrally, we apply production models where the total factor productivity estimates (or the entire technology) are functions of management.

To begin, we use a version of the stochastic frontier model where the mean and the variance of the inefficiency distribution are functions of management. This model assumes neutrality but allows us to investigate monotonicity. Figure 2, plots the marginal effects of management on the mean and variance of the inefficiency distribution against the level of management. The marginal effects are negative but increasing, i.e., better management monotonically decreases the mean and variance of the inefficiency distribution. At the mean the marginal effect on $E(u)$ is -0.17 , similar to the effect for the second stage regression we found above. This result is evidence that when we use estimates of $u$ as proxies for unobserved management,

Fig. 1 Proxies against observed management. Notes: These plots show the lines of linear best fit, the 95\% prediction intervals, and the data for the regressions of fixed effects or time-invariant inefficiency against management and a constant 
Fig. 2 Management as a determinant of the distribution of inefficiency. Notes: The graphs give the marginal effects of management on the mean and variance of the distribution of inefficiency


we can be confident that lower inefficiency always correlates with better management. As an aside, better management also reduces production uncertainty, i.e., for higher levels of management there is less variation in inefficiency. A possible explanation is that better management makes firms more resilient to changes in their environments.

Next, we use two semiparametric models where management shifts the technology not via the inefficiency term but directly via the coefficients. The semiparametric smooth coefficient (SPSC) model allows all coefficients to be nonparametric functions of management. The nested PL model allows only the intercept to be a fully flexible function of management. In both models the parametric part has a Cobb-Douglas form. Again, we control for country and industry fixed effects by mean transforming the variables before estimation, i.e., measurement is in terms of deviations from country-industry means.

We use both semiparametric models to further investigate monotonicity. Unlike a linear parametric model the semiparametric models allow the relation between management and productivity to be different at every point. We test the assumption of monotonicity by plotting the intercept (TFP) estimates from the SPSC and PL models against the management variable in Fig. 3. The solid line is for the SPSC model with the dashed lines giving the bootstrapped 95\% confidence interval. ${ }^{8}$ The confidence interval at the upper end is fairly wide due to a sparsity of observations. Nevertheless, as SPSC is locally weighted it explains the data away from the mean better than parametric models.

\footnotetext{
$\overline{8}$ Appendix A gives details on the bootstrap procedure. We use 100 replications.
}

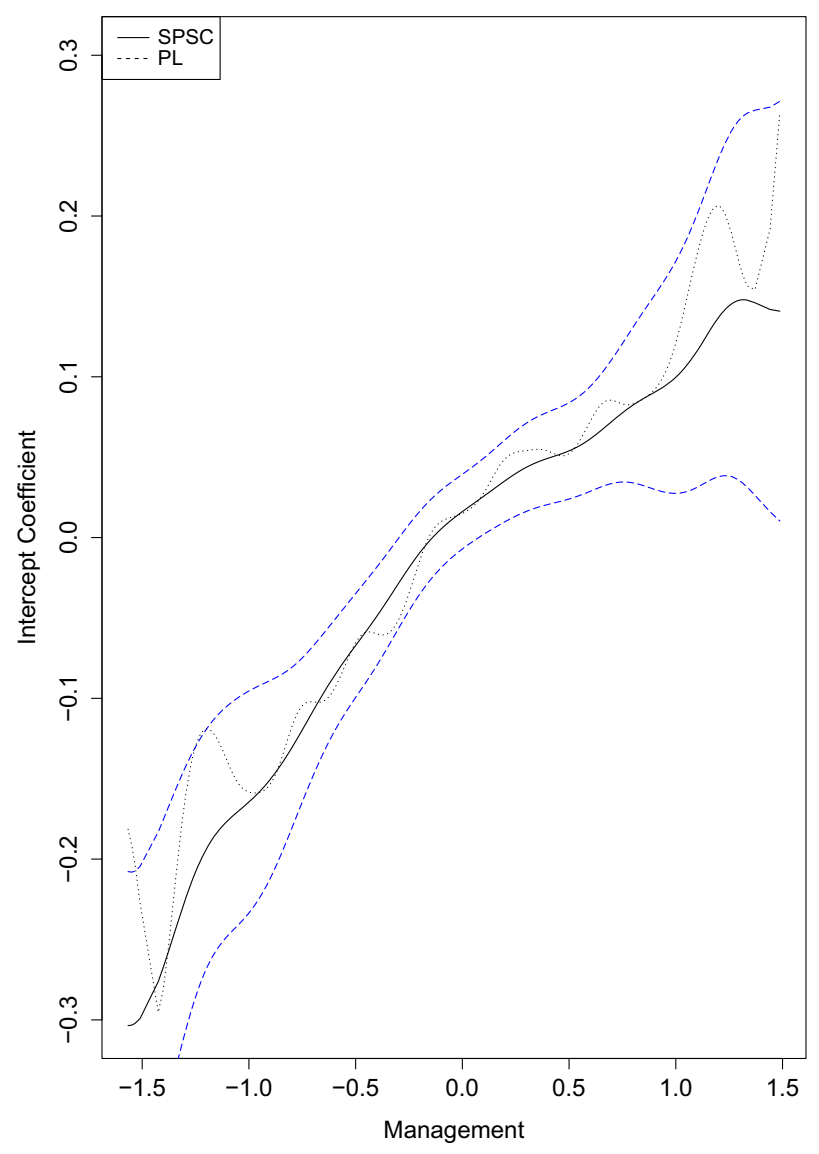

Fig. 3 This graph plots estimated intercept coefficients against management practice for the SPSC (solid) and PL (dotted) models. For the SPSC model it adds the $95 \%$ bootstrapped confidence intervals (dashed lines) 
The specification test proposed by Li and Racine (2010) rejects the Null of the linear parametric model in favor of the SPSC model (the $p$-value is zero to the fourth decimal place $\left.^{9}\right)$. That is, the coefficients are not constant over $z$. We see that TFP is increasing in management everywhere. The assumption that better management always increases productivity seems valid. But what if we restrict management's effect to the intercept, as models that use productivity estimates to measure management do? The dotted line gives the intercept estimates for the PL model. The line fluctuates, which is evidence that TFP is not increasing in management everywhere. However, when testing the Null of a parametric model where management enters linearly against the PL model we cannot reject the linear model. The $p$-value is 0.11 . The comparison with the SPSC model's intercept suggests that the fluctuations are probably not an artifact of the data. We believe that the non-monotonic relation between the PL model's intercept and management suggests that the neutral management channel is not fully independent of the capital and labor channels (discussed below).

To explore the non-neutral effects of management we plot the SPSC model's coefficient estimates for capital and labor in Fig. 4. The two upper panels give the distributions for the two coefficients. Assuming competitive input and output markets these input elasticities reflect revenue shares. Both distributions are fairly wide and probably multimodal. In the lower panels we plot the same coefficients against management together with their $95 \%$ confidence intervals as well as equivalent linear parametric (OLS) estimates. We see that firms with low capital and high labor shares have poor management practices. The opposite is true for firms with high capital and low labor shares. It seems is that the optimal management quality depends on the labor intensity of the firm. Firms with a low labor intensity might employ skilled workers, which require better management practices. Over most of the range of management the constant parametric coefficient estimate falls within the SPSC estimates' confidence intervals. Nevertheless, as already reported above the test of $\mathrm{Li}$ and Racine (2010) rejects the linear parametric model in favor of the SPSC model. As the parametric model is nested in the SPSC model this is evidence that the parametric model is mis-specified ( $\mathrm{Li}$ et al. 2002, p. 417). A formal test for neutrality is to apply the same specification test to the Null of the PL model.${ }^{10}$ For our data, the PL model is rejected in favor of the SPSC model with a $p$-value zero to the fourth decimal place. We conclude that management has important non-neutral effects. Although the question of whether

\footnotetext{
${ }_{9}$ We use a wild cluster bootstrap and 399 replications.

${ }^{10}$ We use a wild cluster bootstrap and 399 replications. For the Null we use the beta coefficients from a OLS regression on the data transformed as suggested by Robinson (1988).
}

management is a technology or an input is not the focus of this paper, our results suggest that it is a bit of both. The fact that total factor productivity is increasing in management supports the view that management is akin to a technology. On the other hand, the finding that management correlates with capital and labor shares suggests that different types of firms have different management practices. To the extent that these differences reflect optimization behavior, management is an input.

As in the SPSC model all coefficients are functions of management, we can correlate characteristics of the production technology, like returns to scale, with management. Figure 5 plots estimates of returns to scale based on the SPSC model, together with their $95 \%$ bootstrapped confidence intervals, against management. For comparison, we also plot the returns to scale estimates from a parametric model (omitting management). The constant parametric estimates fall within the SPSC model's confidence intervals. However, the point estimates differ between the two models, especially for firms with poor management practices. Firms with poor management practices are too small. Above a certain level of management, scale is optimal for a wide range of management practices, but it is not clear which way causality runs.

\section{Conclusion}

Management is an important, but usually unobserved factor in production. Theoretically, management is important, because it is a key mechanism through which owners and regulators try to influence the performance of firms. Economists often use total factor productivity estimates as proxies for management. We take advantage of the management data produced by Bloom and Van Reenen (2007) to assess the empirical usefulness of productivity estimates as proxies for management. First, we correlate two popular proxies, fixed effects and inefficiency scores, with observed management. Second, we use flexible models of production to test whether management influences productivity as assumed by the proxies: neutrally and monotonically.

We find that the correlations between the proxies and management are positive but weak. Also, observed management practices explain only a small fraction, no more than $5 \%$, of the variances of fixed effects and inefficiency scores. This result is no surprise as fixed effects and inefficiency scores are essentially residuals, which capture all factors that are time-invariant or follow an asymmetric distribution, respectively. Overall, fixed effects seem better management proxies than inefficiency scores but require panel data. Of course, our analysis assumes that Bloom and Van Reenen (2007)'s management measure is a good proxy 
(a) Capital (SPSC)

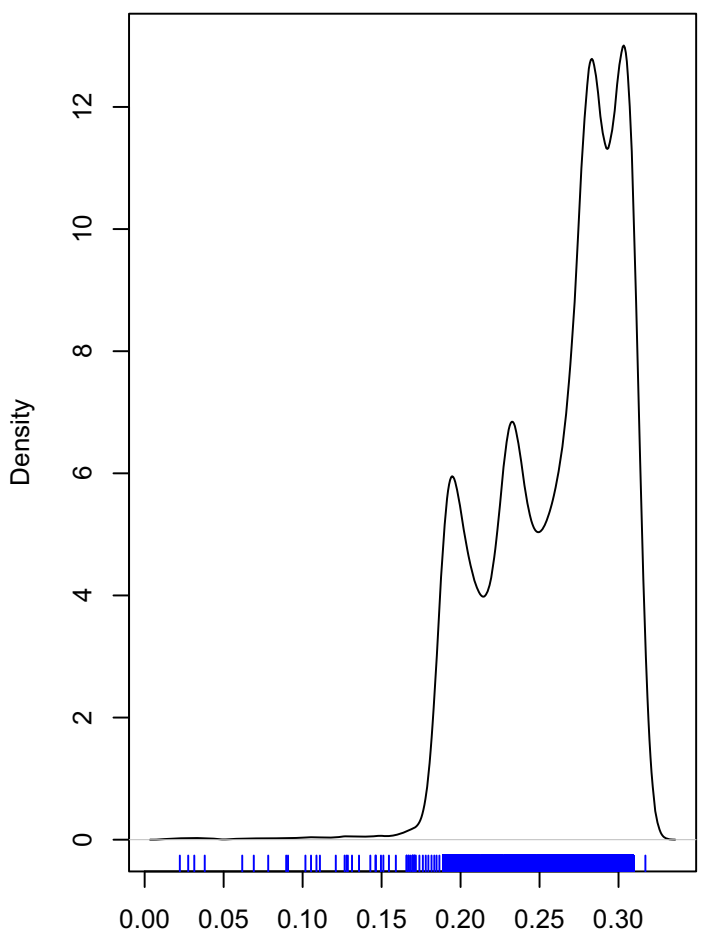

(c) Capital (SPSC)

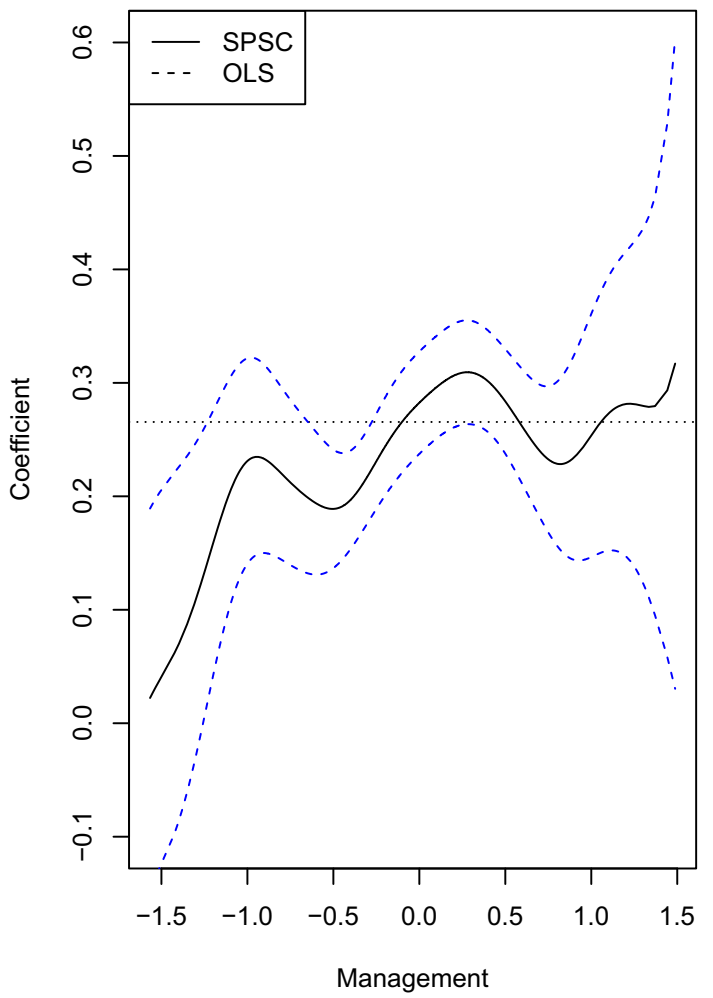

Fig. 4 This graph plots the coefficient estimate for capital and labor from the SPSC model. The upper panels plot the densities of the coefficient estimates. The lower panels plot the coefficient estimates,

\section{(b) Labour (SPSC)}

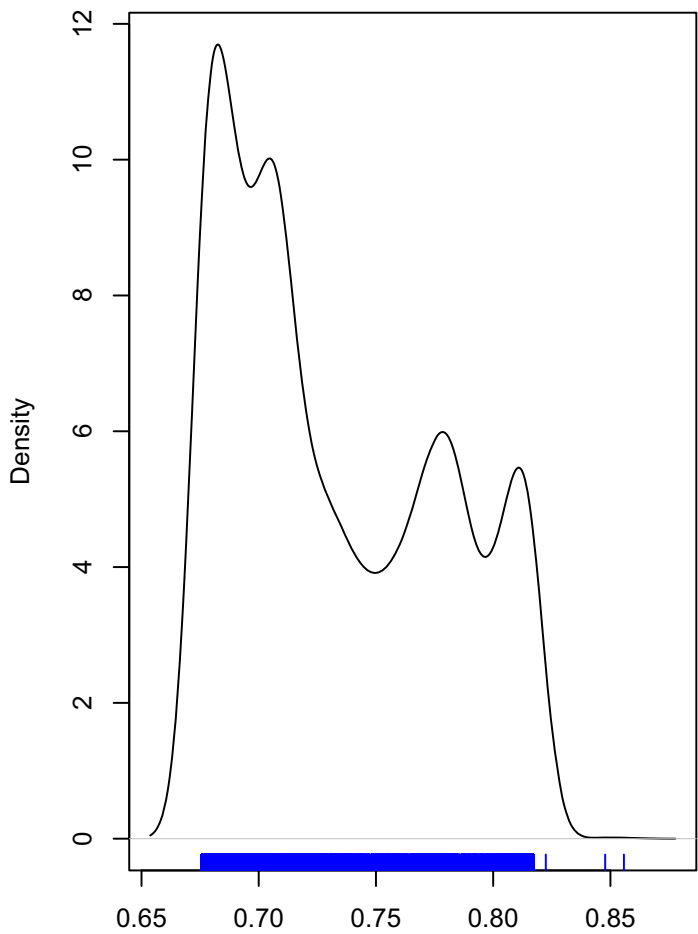

(d) Labour (SPSC)

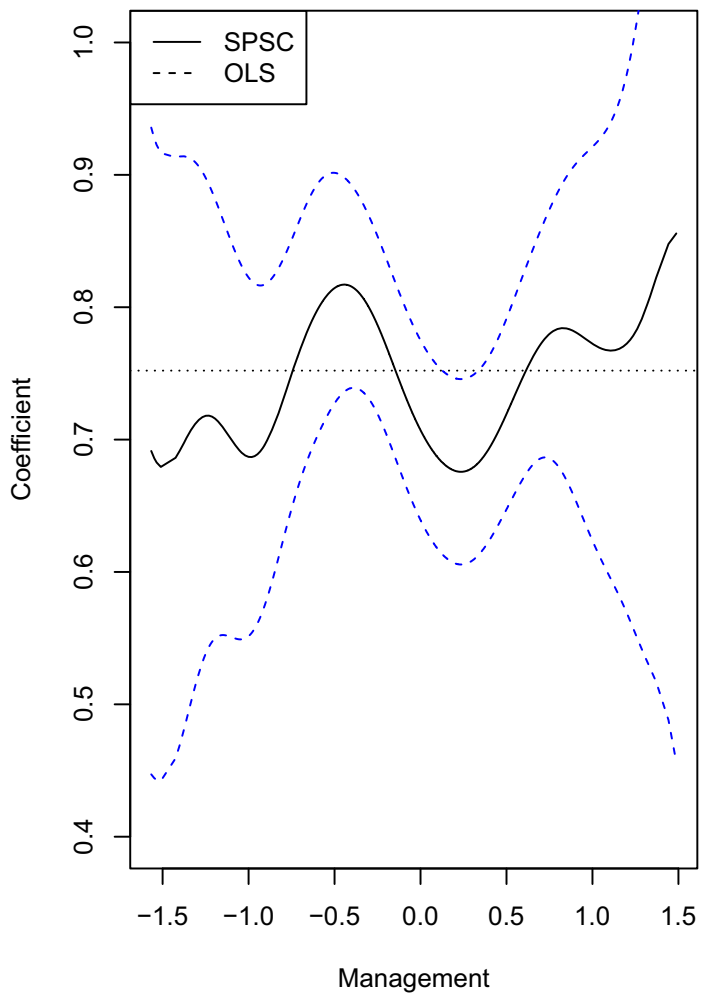

together their $95 \%$ confidence intervals and their equivalent parametric estimates, against management 




Fig. 5 This graph plots returns to scale for the SPSC model against management including the $95 \%$ bootstrapped confidence interval. The dashed line gives the returns to scale for a fully parametric Cobb-Douglas model (omitting management)

itself. There might be dimensions of management quality omitted by their survey measure.

When measuring management by proxies the correlation is of interest but so are the assumptions of neutrality and monotonicity. Testing these assumptions requires models that do not impose them. To test neutrality, we contrast the results from two semiparametric models: one that allows non-neutral effects and another that imposes neutrality. We find that the effect of management is not strictly neutral. Besides total factor productivity, management shifts capital and labor elasticities, too. A formal test also rejects the neutral model in favor of the non-neutral model. We investigate monotonicity by plotting managementdependent total factor productivity estimates from different models. Across, these models TFP estimates are mostly increasing monotonically in management. Last, as management shifts TFP and correlates with the labor-capital ratio it seems that management has characteristics of a technology and an input.

Despite rejecting strict neutrality and also finding some evidence against monotonicity, we believe that overall our evidence supports the use of productivity estimates as proxies for management for some objectives. When the objective is to rank firms by their management abilities the use of the proxies is fine. However, the proxies do not quantify management practices well. For instance, many regulators base price caps on estimates of inefficiency ("benchmarking"). The scope for performance improvement via better management is probably only a fraction of the face value of inefficiency estimates.

Acknowledgements We would like to thank the participants of the 13th European Workshop on Efficiency and Productivity Analysis in Helsinki and the participants of the Eighth North American Productivity Workshop in Ottawa for their useful comments. We would like to thank Hal Fried and Loren Tauer for pointing out the potential of the data used here. We would also like to thank Boris Bravo-Ureta, Kai Sun, Daniel Henderson, Jaap Bos, Mark Sanders, and Mette Asmild for their comments. And we would like to thank two anonymous referees for their useful comments which led to an improved version of the paper. The usual disclaimer applies.

\section{Compliance with ethical standards}

Conflict of interest The authors declare that they have no conflict of interest.

Open Access This article is distributed under the terms of the Creative Commons Attribution 4.0 International License (http://crea tivecommons.org/licenses/by/4.0/), which permits unrestricted use, distribution, and reproduction in any medium, provided you give appropriate credit to the original author(s) and the source, provide a link to the Creative Commons license, and indicate if changes were made.

\section{Appendix}

\subsection{A. Semiparametric estimation}

We estimate (11) using a local linear least-squares estimator proposed by $\mathrm{Li}$ et al. 2002 and $\mathrm{Li}$ and Racine (2010). For this we rewrite (11) as

$Y_{i}=W_{i}^{\prime} \gamma\left(Z_{i}\right)+u_{i}$

then pre-multiply by $W_{i}$ and take expectations conditional on $Z_{i}$, which gives

$$
E\left[W_{i} Y_{i} \mid Z_{i}\right]=E\left[W_{i} W_{i}^{\prime} \mid Z_{i}\right] \gamma\left(Z_{i}\right)+E\left[W_{i} u_{i} \mid Z_{i}\right]
$$

Under the assumption $E\left[W_{i} u_{i} \mid Z_{i}\right]=0$, we can express $\gamma$ (.) as

$$
\gamma\left(Z_{i}\right)=\left(E\left[W_{i} W_{i}^{\prime} \mid Z_{i}\right]\right)^{-1} E\left[W_{i} Y_{i} \mid Z_{i}\right] .
$$


Table 2 The management practice dimensions

Categories Score from 1 to 5 based on

1) Introduction of modern manufacturing techniques

2) Rationale for introduction of modern manufacturing techniques

3) Process problem documentation

4) Performance tracking

5) Performance review

6) Performance dialog

7) Consequence management

8) Target balance

9) Target interconnection

10) Target time horizon

11) Targets are stretching

12) Performance clarity

13) Managing human capital

14) Rewarding high performance

15) Removing poor performers

16) Promoting high performers

17) Attracting human capital

18) Retaining human capital
What aspects of manufacturing have been formally introduced, including just-in-time delivery from suppliers, autonomation, flexible manpower, support systems, attitudes, and behavior?

Were modern manufacturing techniques adopted just because others were using them, or are they linked to meeting business objectives like reducing costs and improving quality?

Are process improvements made only when problems arise, or are they actively sought out for continuous improvement as part of a normal business process?

Is tracking ad hoc and incomplete, or is performance continually tracked and communicated to all staff?

Is performance reviewed infrequently and only on a success/failure scale, or is performance reviewed continually with an expectation of continuous improvement?

In review/performance conversations, to what extent is the purpose, data, agenda, and follow-up steps (like coaching) clear to all parties?

To what extent does failure to achieve agreed objectives carry consequences, which can include retraining or reassignment to other jobs?

Are the goals exclusively financial, or is there a balance of financial and nonfinancial targets?

Are goals based on accounting value, or are they based on shareholder value in a way that works through business units and ultimately is connected to individual performance expectations?

Does top management focus mainly on the short term, or does it visualize short-term targets as a "staircase" toward the main focus on long-term goals?

Are goals too easy to achieve, especially for some "sacred cows" areas of the firm, or are goals demanding but attainable for all parts of the firm?

Are performance measures ill-defined, poorly understood, and private, or are they well-defined, clearly communicated, and made public?

To what extent are senior managers evaluated and held accountable for attracting, retaining, and developing talent throughout the organization?

To what extent are people in the firm rewarded equally irrespective of performance level, or are rewards related to performance and effort?

Are poor performers rarely removed, or are they retrained and/or moved into different roles or out of the company as soon as the weakness is identified?

Are people promoted mainly on the basis of tenure, or does the firm actively identify, develop, and promote its top performers?

Do competitors offer stronger reasons for talented people to join their companies, or does a firm provide a wide range of reasons to encourage talented people to join?

Does the firm do relatively little to retain top talent or do whatever it takes to retain top talent when they look likely to leave?

Note: This table is reproduced from Bloom and Van Reenen (2010)

This formula can be used to estimate $\gamma\left(Z_{i}\right)$. Li and Racine (2010) propose the following local constant estimator

$\widehat{\gamma}(z)=\left[\sum_{j=1}^{n} W_{i} W_{i}^{\prime} K\left(\frac{Z_{j}-z}{h}\right)\right]^{-1} \sum_{j=1}^{n} W_{i} Y_{i} K\left(\frac{Z_{j}-z}{h}\right)$

which is a standard least-squares estimator but for the inclusion of $K(\cdot)$, a diagonal kernel or weighting matrix where the $i$ th element is $K_{i}=K_{h}\left(z_{i}, z\right)$ and $h$ is a vector of bandwidths. The kernel weights the nearby observations. We use a Gaussian kernel. The intuition is that if $K(\cdot)$ was a Gaussian kernel and $z$ was a scalar, $\gamma(z)$ would be a least-squares estimator for $z$ close to $Z$. Generally the bandwidth is obtained by minimizing the squared residuals for the regression curve. We obtain bandwidths using the fully automated least-squares crossvalidation method where

$$
C V(h)=\min _{h} n^{-1} \sum_{i=1}^{n}\left[Y_{i}-W_{i}^{\prime} \widehat{\gamma}_{-i}(Z)\right]^{2} M\left(Z_{i}\right)
$$

where $\widehat{\gamma}_{-i}(z)$ is the leave-one-out estimator and $0 \leq M$ $(\cdot) \leq 1$ a weight function. ${ }^{11}$ This procedure validates the regression curve by its ability to predict out of sample. This makes cross-validation sensitive to outliers (Hartarska et al. 2011). Bloom and Van Reenen (2010, footnote 2) stress that the data we use is noisy. Therefore, we employ the outlier detection method proposed by Filzmoser et al. (2008) and remove about $11 \%$ of the observations; 5629 observations

$\overline{11}$ For details see Li and Racine (2010). 
remain. We obtain estimates for $\beta\left(z_{i}\right)$ for each data point as the $z$ variable is observation specific. Confidence intervals for the coefficients are obtained using a wild cluster bootstrap (Hardle and Mammen 1993). We cluster at the firm level as errors are probably correlated within panels. We conduct our analysis using the np package (Hayfield and Racine 2008) for R (R Development Core Team 2008).

\subsection{B. Survey questions}

Table 2 reproduces the original survey questions.

\section{References}

Aigner D, Lovell CAK, Schmidt P (1977) Formulation and estimation of stochastic frontier production function models. J Econom 6 (1):21-37

Bloom N, Sadun R, Van Reenen J (2017) Management as a technology. CEP Discussion Paper No 1433, London School of Economics and Political Science

Bloom N, Van Reenen J (2006) Measuring and explaining management practices across firms and countries. National Bureau of Economic Research Working Paper Series, No. 12216. National Bureau of Economic Research, Cambridge

Bloom N, Van Reenen J (2007) Measuring and explaining management practices across firms and countries. Q J Econ 122 (4):1351-1408

Bloom N, Van Reenen J (2010) Why do management practices differ across firms and countries? J Econ Perspect 24(1):203-224

Farrell MJ (1957) The measurement of productive efficiency. J R Stat Soc Ser A 120(3):253-290

Filzmoser P, Maronna R, Werner M (2008) Outlier identification in high dimensions. Comput Stat Data Anal 52(3):1694-1711
Hardle W, Mammen E (1993) Comparing nonparametric versus parametric regression fits. Ann Stat 21(4):1926-1947

Hartarska V, Parmeter CF, Nadolnyak DA (2011) Economies of scope of lending and mobilizing deposits in microfinance institutions: A semiparametric analysis. Am J Agric Econ 93(2):389-398

Hayfield T, Racine JS (2008) Nonparametric econometrics: The np package. J Stat Softw 27(5):1-32

Hsiao C, Li Q, Racine JS (2007) A consistent model specification test with mixed discrete and continuous data. J Econom 140 (2):802-826

Jondrow J, Knox Lovell CA, Materov IS, Schmidt P (1982) On the estimation of technical inefficiency in the stochastic frontier production function model. J Econom 19(2-3):233-238

Li Q, Huang CJ, Li D, Fu TT (2002) Semiparametric smooth coefficient models. J Bus Econ Stat 20(3):412-422

Li Q, Racine JS (2010) Smooth varying-coefficient estimation and inference for qualitative and quantitative data. Econom Theory 26 (06): 1607-1637

Meeusen W, van den Broeck J (1977) Efficiency estimation of Cobb-Douglas production functions with composed error. Int Econ Rev 18:435-444

Mundlak Y (1961) Empirical production function free of management bias. J Farm Econ 43(1):44-56

Nickell SJ (1996) Competition and corporate performance. J Political Econ 104:724-746. 4

Nuthall P (2009) Modelling the origins of managerial ability in agricultural production. Aust J Agric Resour Econ 53(3):413-436

R Development Core Team (2008) R: a language and environment for statistical computing. R Foundation for Statistical Computing, Vienna

Robinson PM (1988) Root-n-consistent semiparametric regression. Econometrica 56(4):931-954

Wang H-J (2002) Heteroscedasticity and non-monotonic efficiency effects of a stochastic frontier model. J Product Anal 18(3): $241-253$ 